\title{
Manifestation of Marine Lenhart Syndrome after failed Radioactive Iodine therapy
}

\author{
Francis Essien ${ }^{1}$, Callie Cheatham ${ }^{1}$, Blake Elkins ${ }^{1}$, and Joshua Tate ${ }^{1}$ \\ ${ }^{1}$ Keesler Air Force Base
}

April 6, 2021

\begin{abstract}
Marine Lenhart Syndrome (MLS) is caused by a coexistence of active thyroid nodules and Graves' disease1. Here, we present a case of hyperthyroidism characterized by the presence of stimulating TSH receptor antibodies, unsuccessful radioactive iodine ablation, ultimately requiring Methimazole followed by thyroidectomy. We review the current literature.
\end{abstract}

Title: Manifestation of Marine Lenhart Syndrome after failed Radioactive Iodine therapy

Authors: Essien, Francis ${ }^{1}$; Cheatham, Callie ${ }^{1}$; Elkins, Blake ${ }^{1,2}$; Tate, Joshua ${ }^{1,2}$

Author Affiliations:

${ }^{1}$ Keesler Air Force Base, MS; Keesler Medical Center, Department of Internal Medicine

${ }^{2}$ Keesler Air Force Base, MS; Keesler Medical Center, Department of Internal Medicine, Division of Endocrinology

Background/Objective:

Primary hyperthyroidism is the result of overproduction of thyroid hormone resulting in the classic symptoms of tachycardia, weight loss, diaphoresis, and hyperdefecation. There are multiple common causes to include Graves' disease, toxic multinodular goiter, and solitary toxic adenomas. Marine Lenhart Syndrome (MLS) is a rare cause of hyperthyroidism, caused by a coexistence of constitutively active thyroid nodules and Graves' disease $^{1}$. The prevalence of this syndrome has been reported to be between $2.7-4.1 \%^{2}$, though is likely an under recognized cause of hyperthyroidism. We present a rare case of MLS unmasked following radioactive iodine (RAI) ablation along with a review of the literature.

Case Report:

A 60 year old African American female was referred to endocrinology clinic in October 2016 for hyperthyroidism indicated by a suppressed TSH and an elevated free thyroxine (FT4) of $4.9 \mathrm{ng} / \mathrm{dL}$ (reference range $0.76-1.46 \mathrm{ng} / \mathrm{dL}$ ). She was also noted to have the following associated findings: six month 80lb weight loss, hyperdefecation, agitation, palpitations, and newly diagnosed atrial fibrillation with rapid ventricular rate. Physical exam was notable for diffuse thyroid enlargement and mild bilateral eyelid edema. Repeat labs confirmed biochemical hyperthyroidism and also noted elevated thyroid stimulating immunoglobulin (TSI) at $437 \%$ (reference range $<139 \%$ ). Thyroid ultrasound (US) was obtained and demonstrated multiple nodules bilaterally with increased vascularity in the left lobe (Figure 1).

In the setting of biochemical evidence of hyperthyroidism, elevated TSI, and signs/symptoms of thyrotoxicosis, the patient was diagnosed with Graves' disease and she opted for RAI ablation. Prior to ablation, she underwent radioactive iodine uptake (RAIU) and scan to further evaluate the thyroid nodules and for 
iodine-123 treatment dose calculation per institutional protocol. Thyroid scan revealed diffuse thyroid uptake consistent with Graves' disease with a left inferior hypoactive (cold) area that corresponded to a cystic nodule previously noted on ultrasound (Figure 2). Biopsies of the nodule were obtained and benign. She underwent RAI ablation with 31.3mCi in January 2017.

At follow up in April 2017, the patient was asymptomatic but continued to have labs indicating hyperthyroidism. As a result, repeat RAI ablation was planned but a repeat RAIU and scan was obtained for RAI ablation dose calculation per institutional protocol. The repeat scan demonstrated a toxic multinodular goiter with increased uptake of $47.9 \%$ (Figure 3). At this time, the patient opted for total thyroidectomy for definitive therapy, which occurred in May 2017 with benign pathology.

Discussion:

Primary hyperthyroidism is caused by excess thyroid hormone production which can present with associated clinical findings such as tachycardia, tremor, heat intolerance, hyperdefecation, and weight loss. Laboratory evaluation is typically notable for a low TSH and an elevated FT4. Primary hyperthyroidism is most commonly due to Graves' disease, accounting for $60-80 \%$ of hyperthyroid cases. Graves' disease is characterized by serum antibodies against TSH receptors in correspondence with T cell mediated immunity. Toxic adenomas or toxic multinodular goiters, also known as Plummer's disease, are a result of several somatic point mutations in the third trans-membrane loop of the TSH receptor leading to autonomous function in absence of $\mathrm{TSH}^{3}$. Though both disease processes result in thyrotoxicosis, their mechanism of actions are distinct.

The presence of thyroid nodules coincide with Graves' disease in $25-30 \%$ of patients ${ }^{2}$. Though most of these nodules are hypo-functioning, as initially thought to be the case above, rare cases are noted to be hyperactive. The term Marine Lenhart syndrome (MLS) has been assigned to this presentation with the coexistence of Graves' disease and toxic adenomas and has become more frequently recognized within the literature ${ }^{1}$. However, there is controversy regarding formal diagnosis due to variations in imaging techniques, presentation, and onset ${ }^{4}$. The definition and diagnostic criteria for Marine Lenhart Syndrome have subsequently undergone multiple revisions.

The syndrome as described in 1972 by David Charkes ${ }^{5}$ based off the original 1911 observations of Marine and Lenhart ${ }^{6}$ was defined as patients with Graves' disease and toxic adenomas that exhibit increased radioiodine uptake with TSH stimulation, appear hypofunctioning in relation to extranodular tissue on RAIU and scan, are poorly responsive to RAI ablation, and exhibit increased radioiodine uptake after RAI ablation therapy ${ }^{5}$. Thus, one of the key distinctions of the toxic adenomas of MLS compared to those autonomous nodules found in Plummer's disease was their TSH dependence. A recent literature review in 2011 by Biersack suggested that MLS may be a conjoined syndrome; the classical Graves' disease/toxic adenoma vs Graves' disease post RAI ablation with autonomously functioning thyroid nodules ${ }^{1}$. Examination of the previous definition of toxic adenomas by Plummer defines hyperthyroidism as a disease caused by two separate entities (Toxic adenoma and exophthalmic goiter $)^{1}$. However the text by Marine in 1940 failed to appropriately distinguish these two conditions noting toxic adenoma and immunogenic goiter as one condition. Thus the original term may not apply to what we now know to be two separate diseases and the medical literature needs to be updated to reflect the origin of the joint presentation.

Recently documented cases of MLS include cases of coexisting Graves' disease and hyperfunctioning nodules at the time of diagnosis ${ }^{7-10}$, cases of Graves' disease with "cold" nodules at the time of diagnosis that then later were confirmed as hyperfunctioning ${ }^{11}$, and cases of development of toxic adenomas years after successful treatment of Graves' disease ${ }^{12-13}$. This highlights the need for a unified definition of MLS, which was proposed by Neuman et al in $2018^{7}$. These proposed diagnostic criteria included confirmed hyperthyroidism based on thyroid function tests with positive thyroid autoantibodies consistent with Graves' disease, RAIU scan revealing hyperfunctioning nodules that correspond to nodules seen on ultrasound on a background of diffusely increased radioiodine uptake, and confirmation of the presence of a follicular adenoma or hyperplastic lesion on pathologic analysis. Pathologic analysis is important as there have been 5 cases reported in the literature thus far of MLS patients with the additional finding of papillary thyroid carcinoma identified 
in an associated nodule with the most recent being published by Mehmet et al of a unique case of papillary thyroid carcinoma in a hyper functioning nodule ${ }^{14}$. This definition also encouraged a transition from using the term "autonomous" nodules to describing them as functional nodules to include the subset of MLS cases with apparent TSH dependence of the associated nodules. In fact, prior proposed definitions included the requirement for the associated nodules to demonstrate TSH dependence ${ }^{15}$. This currently proposed diagnostic criteria allows for inclusion of the multiple identified variants of MLS to include this unique case of nodules that appeared cold on initial RAIU scan but were later confirmed as hyperfunctioning nodules on subsequent scans after completion of RAI ablation without evidence of return to a chemically euthyroid state.

Conclusion:

Marine Lenhart Syndrome remains a rare and poorly defined entity that should be considered different both diagnostically and therapeutically from any etiology of thyrotoxicosis in isolation. In the presented case, the patient was noted to have a toxic multinodular goiter after completion of RAI ablation for previously diagnosed Graves' disease without returning to a euthyroid baseline. She subsequently underwent total thyroidectomy, which remains the preferred therapy for MLS. A broader definition and increased awareness is needed to allow for better understanding and improved treatment guidelines for this syndrome. Additionally, this case highlights the importance of considering Marine Lenhart Syndrome in any patient being treated for Graves' disease who has known thyroid nodules and does not respond to RAI ablation.

The authors confirm contribution to the paper as follows:

Study conception and design: Francis Essien D.O., Joshua Tate M.D.,

Data collection: Francis Essien D.O., Joshua Tate M.D. analysis and interpretation of results: Francis Essien D.O., Joshua Tate M.D., Blake Elkins M.D., Callie Cheatham M.D.

Draft manuscript preparation: Francis Essien D.O., Joshua Tate M.D., Blake Elkins M.D.

All authors discussed the results and contributed to the final manuscript.

References:

1. Biersack, H., Biermann, K. The Marine Lenhart Syndrome Revisited. The Central European Journal of Medicine. 2011. 123: 459-462

2. Cakir, M. Marine Lenhart Syndrome. Journal of the national medical association. 2005. 97: 7

3. Avci, E., Narci, H. Coexistence of Graves' disease and toxic adenoma: a rare presentation of MarineLenhart Syndrome. J Ayub Med Coll Abbottabad. 2015. 27:1

4. Brahma et al. The Oldest case of Marine-Lenhart syndrome? 2012. Journal of the Royal Society of Medicine short reports. 3:21

5. N. D. Charkes, "Graves' disease with functioning nodules (Marine-Lenhart syndrome).," Journal of Nuclear Medicine, vol. 13, no. 12, pp. 885-892, 1972.

6. Marine D, Lenhart CH. Pathological Anatomy of Exophalmic goiter - The anatomical and physiological relations of the thyroid gland to the disease; the treatment. Arch Int Med 1911;8:265-316.

7. Cakir M. Marine-Lenhart syndrome. J Natl Med Assoc . 2005;97(7):1036-1038.

8. Danielle Neuman, Russ Kuker, Francesco Vendrame, "Marine-Lenhart Syndrome: Case Report, Diagnosis, and Management", Case Reports in Endocrinology, vol. 2018, Article ID 3268010, 4 pages, 2018.

9. Scherer T, Wohlschlaeger-Krenn E, Bayerle-Eder M, et al. A Case of simultaneous occurrence of Marine - Lenhart syndrome and a papillary thyroid microcarcinoma. BMC Endocr Disord . 2013;13:16. Published 2013 May 8. doi:10.1186/1472-6823-13-16

10. Miyazaki M, Okada Y, Torimoto K, Tanaka Y. A Case of Marine-Lenhart Syndrome with Predominance of Plummer Disease. J UOEH . 2019;41(2):165-170. doi:10.7888/juoeh.41.165

11. Brahma A, Beadsmoore C, Dhatariya K. The oldest case of Marine-Lenhart syndrome?. JRSM Short Rep . 2012;3(4):21. doi:10.1258/shorts.2011.011164

12. Waldherr C, Otte A, Haldemann A, Müller-Brand J. Das Marine-Lenhart-Syndrom: Eine Fallbeobachtung über 18 Jahre [Marine-Lenhart syndrome: a case observation upon 18 years]. Nuklearmedizin . 
$1999 ; 38(8): 345-348$.

13. Giuffrida G, Giovinazzo S, Certo R, et al. An uncommon case of Marine-Lenhart syndrome. Arq Bras Endocrinol Metabol . 2014;58(4):398-401. doi:10.1590/0004-2730000003173

14. Mehmet et al. A Rare Presentation of Autonomously Functioning Papillary Thyroid Cancer: Malignancy in Marine-Lenhart Syndrome Nodule. 2016. Case Reports in Surgery.

15. Harisankar CN, Preethi GR, Chungath BB. Hybrid SPECT/CT evaluation of Marine-Lenhart syndrome. Clin Nucl Med . 2013;38(2):e89-e90. doi:10.1097/RLU.0b013e31825ae860

\section{Hosted file}

MLS Images_JT Edits.pdf available at https://authorea.com/users/405924/articles/516819manifestation-of-marine-lenhart-syndrome-after-failed-radioactive-iodine-therapy 